\title{
Metodologías didácticas para el aprendizaje en línea
}

\author{
Vicente Gabarda Méndez - Universidad Internacional de Valencia \\ (iD) 0000-0001-6159-5173 \\ Ernesto Colomo Magaña - Universidad Internacional de Valencia \\ (iD) $0000-0002-3527-7937$ \\ Mercedes Romero Rodrigo - Universidad Internacional de Valencia \\ (iD) $0000-0002-9103-5668$
}

Recepción: 24/01/2019 | Aceptado: 06/03/2019

Correspondencia a través de ORCID: Vicente Gabarda

0000-0001-6159-5173

Citar: Gabarda, V, Colomo, E y Romero, MM (2019). Metodologías didácticas para el aprendizaje en línea. REIDOCREA, 8(2), 19-36.

\begin{abstract}
Resumen: La modalidad online de enseñanza y aprendizaje ha permitido deslocalizar y romper con la barrera temporal tradicional de los procesos formativos. Esta modalidad, aunque se está consolidando de manera fehaciente en diferentes contextos, sigue en continuo cambio debido a los avances tanto tecnológicos como sociales que confluyen en su utilización. Dichos cambios, que afectan de manera directa al desarrollo de nuevos paradigmas y nuevas herramientas, están permitiendo un avance exponencial en el modo en que se diseñan y llevan a cabo los procesos de enseñanza aprendizaje. A través de las herramientas tecnológicas, y siempre en coherencia con el desarrollo innegable de la competencia digital del profesorado, es posible plantear propuestas en nuevos espacios (entornos virtuales de aprendizaje) que aportan múltiples posibilidades para el aprendizaje de los estudiantes. En este sentido, el presente ensayo trata de poner de manifiesto las potencialidades del modelo de enseñanza online como facilitador del aprendizaje en la Educación Universitaria. Asimismo, se analizan diferentes propuestas metodológicas para el fomento del aprendizaje colaborativo, el aprendizaje significativo y el aprendizaje autónomo.
\end{abstract}

Palabras clave: Educación universitaria | enseñanza-aprendizaje en línea

\section{Didactic methods for online learning}

Abstract: The online mode of teaching and learning has allowed offshore and break the traditional and temporary barrier that characterizes the training processes. This mode, although it is consolidating in different contexts, is still changing because of the technological and social advances that come together in their use. Those changes, that have a direct influence in the development of new paradigms and new tools, are allowing an exponential progress in the way that the learning and teaching processes are designed and carried out. Through the technological tools, and always consistent with the undeniable development of the digital competence of teachers, it is possible to propose new spaces (virtual learning environments), which provide possibilities for the students learning. In this sense, this essay tries to demonstrate the potential of the online teaching model as a learning facilitator in University Education. It is also analysed different methodological proposals for the promotion of collaborative learning, meaningful learning and independent learning.

Keywords: Higher education | online teaching-learning

\section{Entornos virtuales y formación en línea}

La evolución de los escenarios de eLearning ha sido constante desde su aparición, pasando de una concepción centrada en la figura del docente a un constructo en el que el conocimiento es elaborado de forma colaborativa, donde el alumnado debe adquirir la competencia de aprender a aprender a la vez que el profesorado precisa reaprender a enseñar (Rodríguez, 2003). El auténtico reto en la actualidad es conjugar de forma equilibrada los contenidos de las materias, la didáctica y las tecnologías en un contexto de aprendizaje enriquecedor (Gómez, 2017). En este sentido, fue la propia Organización de las Naciones Unidas para la Educación, la Ciencia y la Cultura (en inglés United Nations Educational, Scientific and Cultural Organization, UNESCO) en la Conferencia Mundial de Educación Superior celebrada en París (2012), quien subrayó el potencial de las tecnologías para convertirse en el canal idóneo para renovar la Educación Superior y facilitar el acceso universal al conocimiento y la información. En este sentido, 
no podemos obviar que esta revolución se está acelerando debido a los continuos avances que se producen en las comunicaciones digitales globales y la mejora potencial de la aplicabilidad de las tecnologías al ámbito educativo (Rodríguez, 2010). En lo que concierne al proceso de enseñanza, el principal cambio del aprendizaje presencial al virtual reside en el potencial comunicativo que los distintos canales de interacción permiten al alumnado, donde factores limitantes como el espacio y el tiempo desaparecen para dar lugar a procesos flexibles, deslocalizados y asíncronos (Coll y Monereo, 2008) en los que se comparten contenidos, reflexiones, dudas, propuestas y se construye de forma colectiva el conocimiento de forma crítica (Rodríguez, 2014; Ruiz, 2016). Esta nueva realidad conlleva implícitamente cambios en los procesos de aprendizaje que se ven modificados respecto a su estructura tradicional, generándose un espacio donde se puede aprender "desde cualquier lugar, en cualquier momento y desde cualquier dispositivo" (Pablo, 2017, p. 44).

De esta premisa surge el modelo de enseñanza online, encargado de dar respuesta a la nueva realidad social donde las tecnologías se convierten en un medio y catalizador (Rodríguez, 2010) para abordar de forma global y holística los procesos formativos de forma eficiente y eficaz sin perder la calidad respecto a la ejecución de los mismos, siendo el diseño del proceso de enseñanza lo que determina el aprendizaje y donde se deben tener en cuenta las posibilidades que ofrecen las tecnologías (Jonassen, Howland, Marra y Crismond, 2008) y los resultados positivos que se alcanzan con estas respecto al aprendizaje tradicional (Bernard, Borokhovski, Schmid, Tamim y Abrami, 2014). De esta manera, los entornos virtuales de aprendizaje se convierten en espacios informáticos con finalidades formativas que favorecen la interacción para el desarrollo de los procesos de enseñanza, con modelos más abiertos y participativos, en los que se incluyen herramientas "para la comunicación sincrónica y asincrónica; para la gestión de los materiales de aprendizaje; para la gestión de las personas participantes, incluidos sistemas de seguimiento y evaluación del progreso de los estudiantes" (Silva y Romero, 2014 , p. 7). Se trata de una nueva vía formativa constituida por características tanto de la presencial como de la educación a distancia, conformando un híbrido que atiende a las necesidades de ambos públicos de manera conjunta. Para ello, surgen diferentes metodologías y herramientas (Durán y Estay, 2016) que permiten el análisis y discusión de los contenidos disciplinares mediante procesos interactivos de forma presencial (videoconferencias, chat interactivo, role playing, etc.) y offline (foros, portfolio digital, correos, podcast, píldoras formativas, etc.). Ha sido la continua evolución de las herramientas e instrumentos la que ha permitido convertir en realidad todas aquellas propuestas didácticas que el profesorado desee implementar en los entornos virtuales de aprendizaje (Johnson et al., 2016), apostando por procesos continuos de innovación y transformación de las prácticas educativas en las que el sentido pedagógico lo da el profesorado (Silva y Romero, 2014).

Para lograr esto hay que reflexionar sobre dos cuestiones claves: dotación y formación. En primer lugar, es necesario disponer de todos aquellos recursos tecnológicos para poder implementar los procesos educativos que se han definido, evitando que exista una reciprocidad negativa entre los indicadores propuestos a alcanzar y la dotación tecnológica real para acometer las acciones pertinentes. En segundo lugar, aparece la necesidad explícita de planes formativos. Y es que siguiendo a Westbrook (2006), la efectividad de la formación en línea va arraigada a la correcta formación y apoyo tanto de los agentes que intervienen en el proceso (docentes y estudiantes), desarrollando estrategias que permitan el uso adecuado de las tecnologías. Mención especial tiene esta formación para el profesorado (Gómez, 2017), quien debe asumir un cambio de rol tanto en el ejercicio de su labor respecto a la praxis docente como en el fomento activo de interacciones que favorezcan el aprendizaje entre el alumnado. De esta manera, incorporar las tecnologías a los procesos educativos conlleva "un cambio sistémico en las formas en que el docente se comunica, piensa y practica su profesión, lo que 
conlleva un reto implícito en su quehacer educativo" (Orozco, Cabezas, Martínez y Alonso, 2016, p. 984). Para un correcto desempeño en los entornos virtuales, se hace preciso adquirir las competencias digitales necesarias para poder desarrollar los procesos formativos en los nuevos espacios virtuales de aprendizaje, ya que el profesorado no puede enseñar aquello que no domina. En este sentido, Owens (2012) denota que el desarrollo de formación sobre entornos formativos online beneficia a la eficacia de la labor docente, así como a la satisfacción con su desempeño profesional. Además, Durán, Gutiérrez y Prendes (2016) demostraron que el nivel de desempeño de la competencia digital en el alumnado estaba determinado por el dominio y control que demuestre el profesorado, de ahí la importancia de asegurarnos que adquieran las destrezas y habilidades precisas.

Todos estos cambios nos llevan a una doble reflexión: por un lado, conocer cómo está actuando el sistema educativo para formar al futuro profesorado en estos espacios de aprendizaje mediados por la tecnología (Castaño, Poy, Tomsa, Flores y Jenaro, 2015); y por otro lado, profundizar en cómo aprende el alumnado en dichos entornos, lo que conlleva repensar el hecho educativo, el ejercicio docente y los estilos de aprendizaje en un contexto que demanda una nueva forma de funcionar y estructurar los procesos (Falco, 2017). Y es que este contexto conlleva un cambio en los roles de los actores del proceso educativo, donde tanto el alumnado como el profesorado ven transformada su realidad.

En el caso del alumnado, pasan a tener un papel activo en el proceso de adquisición de los conocimientos y desarrollo de las competencias, participando de forma íntegra en las experiencias de aprendizaje que tendrán un carácter más dinámico. En este sentido, las TIC se convierten en un medio para la actualización continua de forma autónoma respecto a los procesos de enseñanza-aprendizaje (Ruiz, Sánchez y Gómez, 2013). Es preciso ser conscientes de que estamos en una época en la que las tecnologías han inundado la realidad de todas las generaciones, por lo que es preciso implementar todos estos medios y recursos como elementos presentes en los procesos de aprendizaje (Gómez, 2017).

En lo que respecta al profesorado, su papel pasa a ser el de facilitador y guía, donde lo importante es enseñar cómo aprender y no qué aprender (Castaño, Jenaro y Flores, 2017), reconstruyendo así su identidad docente (Comas-Quinn, 2011). Por tanto, el foco se sitúa más en el aprendizaje y menos en el proceso de enseñar, encargándose de aportar directrices sobre cómo abordar los contenidos, cuáles son los ejes que conforman la materia y las principales preguntas que deben poder responder tras la adquisición de las competencias y conocimientos (Rodríguez, 2010). En este sentido, cobra también relevancia su papel respecto a la planificación, organización, ritmos, estilos y estrategias de aprendizaje, estipulación de criterios y estándares de consecución, así como el desarrollo del proceso evaluativo (García, Luna, Ponce, Cisneros, Cordero y Espinosa, 2018). Además, debe cuidar que el factor humano, tangible en las interacciones que se producen con el alumnado, esté presente en los procesos virtuales de aprendizaje, ya que los estudios denotan que si el factor humano no es protagonista es complejo que los modelos de formación se mantengan vigentes (Armellini y Aiyegbayo, 2010).

Ante esta nueva realidad, existen diferentes cuestiones interesantes sobre las que profundizar: ¿qué recursos tienen más impacto en los procesos de aprendizaje en los entornos virtuales? ¿cómo impactan los entornos virtuales en los diferentes tipos de aprendizaje? 
Para poder abordar todas estas cuestiones, profundizaremos en cómo se están trabajando estos aspectos desde la Universidad Internacional de Valencia, una entidad concebida para desarrollar la formación e-learning adaptándose a las nuevas realidades y necesidades de la sociedad digital.

\section{La universidad Internacional de Valencia: un estudio de caso de formación en línea}

La Universidad Internacional de Valencia es una entidad de educación superior de carácter privado y vocación internacional. Su oferta educativa, que incluye Grados y Postgrados (tanto oficiales como títulos propios), dando respuesta a formación inicial y continua de los profesionales de diversas áreas de conocimiento (Educación, Salud, Empresa, Jurídico, Artes, Humanidades, Comunicación, Ciencia y Tecnología).

Su propuesta formativa se fundamenta en los principios más sólidos de la formación tradicional, pero integrando todo el potencial que aportan los avances tecnológicos. Tal y como apuntábamos anteriormente, la integración de las Tecnologías de la Información y la Comunicación (en adelante, TIC) permite romper los tradicionales esquemas espaciotemporales de los procesos formativos. Se eliminan, así, "los obstáculos que un horario y un lugar físico pueden generar, favoreciendo que los estudiantes tengan potestad para organizar su propio ritmo de aprendizaje" (Colomo, Motos, Aguilar y Gabarda, 2018, p.2878).

En el contexto de esta deslocalización del proceso formativo, esta propuesta es posible a través de un modelo interactivo y multiplataforma, que favorecen un aprendizaje ubicuo (Vázquez y Sevillano, 2015). Tanto las herramientas que fundamentan el aprendizaje desde un punto de vista asíncrono, como las propias sesiones en tiempo real, pueden seguirse a través de cualquier dispositivo móvil, posibilitando la interacción entre profesor y estudiante y entre el propio alumnado.

\section{El modelo académico de la Universidad Internacional de Valencia}

El modelo académico que vertebra el conjunto de procesos de enseñanza y aprendizaje de la institución es, por naturaleza, e-presencial y síncrono. Estos dos conceptos (epresencialidad y sincronía) se materializan a través de todas aquellas sesiones de diferente tipo (clases expositivas, actividades prácticas, tutorías y pruebas objetivas) donde profesorado y alumnado comparten un espacio y un tiempo determinado.

Por otro lado, estas sesiones no solamente proporcionan espacios de encuentro entre alumnado y profesorado, sino que permiten fomentar el aprendizaje colaborativo, creando grupos de trabajo en las propias sesiones. Este tipo de aprendizaje se puede trabajar, complementariamente, a través de otras herramientas de participación asíncrona como los foros o las wikis, que forman parte de las potencialidades del sistema Learning Management System (LMS) que se utiliza como plataforma virtual. En ella, se integran, además, otros recursos y herramientas que permiten atender de un modo integral, y a la vez individualizado, a cada estudiante. 
Focalizando la atención sobre las interacciones en tiempo real, el modelo contempla diferentes tipos de sesiones de trabajo, con fines diversos (ver tabla 1):

Tabla 1. Tipos de sesiones síncronas y fines

\begin{tabular}{|c|l|}
\hline $\begin{array}{c}\text { Clases } \\
\text { expositivas }\end{array}$ & $\begin{array}{l}\text { Se trata de sesiones donde el profesorado, a través de metodologías como } \\
\text { la lección magistral o la lección magistral participativa, expone los } \\
\text { fundamentos teóricos de la asignatura. }\end{array}$ \\
\hline $\begin{array}{c}\text { Clases } \\
\text { prácticas }\end{array}$ & $\begin{array}{l}\text { Son sesiones de trabajo activo por parte del alumnado, que suelen tener } \\
\text { como base del trabajo los fundamentos teóricos vistos en las clases } \\
\text { expositivas. Pueden tener matices diversos en función de aspectos como } \\
\text { las metodologías utilizadas (estudio de casos, resolución de problemas, } \\
\text { revisiones bibliográficas, simulaciones, trabajo cooperativo, entre otras), } \\
\text { los recursos en que se fundamenten (materiales escritos, recursos } \\
\text { audiovisuales, etc.) o los trabajos que se desprenden de estas sesiones y } \\
\text { que formarán parte del portafolio. }\end{array}$ \\
\hline Tutorías & $\begin{array}{l}\text { Las tutorías son espacios síncronos donde se ofrece información de } \\
\text { carácter general, se resuelven dudas y se dan orientaciones específicas } \\
\text { ante dificultades concretas. Pueden tener dos modalidades: tutorías } \\
\text { colectivas (inicio y fin de las asignaturas), que sirven para presentar las } \\
\text { características básicas de organización y funcionamiento de las } \\
\text { asignaturas (inicio), así como para poder valorar y proponer mejoras (fin) y } \\
\text { tutorías individuales, donde alumnado y profesorado comparten } \\
\text { información acerca del progreso académico. }\end{array}$ \\
\hline Pruebas & $\begin{array}{l}\text { Como parte de la evaluación de cada una de las asignaturas (a excepción } \\
\text { de las prácticas y el Trabajo fin de título), se realiza una prueba. Esta } \\
\text { prueba se realiza en tiempo real y tiene como objetivo evidenciar el nivel } \\
\text { de adquisición de conocimientos y desarrollo de competencias por parte } \\
\text { del alumnado. }\end{array}$ \\
\hline
\end{tabular}

Por otro lado, y en sincronía con las especificaciones del Espacio Europeo de Educación Superior (EEES), el trabajo autónomo del alumnado constituye un elemento clave del desarrollo de los procesos formativos. En este sentido, la propuesta de la Universidad Internacional de Valencia contempla que este tipo de actividad puede representar hasta un $60 \%$ de la carga lectiva en el contexto de una asignatura tipo.

En relación con las metodologías utilizadas en el marco del modelo académico, pueden variar según la naturaleza de cada asignatura, seleccionándose las más adecuadas para el desarrollo de las competencias por parte del alumnado. No obstante, se detallan a continuación (ver Tabla 2) algunos ejemplos de metodologías que pueden asociarse a las diferentes actividades formativas de clases expositivas y clases prácticas. 
Tabla 2. Actividades formativas, metodologías y finalidad

\begin{tabular}{|c|c|c|}
\hline Actividad & Metodología & Finalidad \\
\hline \multirow[t]{2}{*}{$\begin{array}{l}\text { Clases } \\
\text { expositivas }\end{array}$} & Lección magistral & $\begin{array}{l}\text { El profesorado presenta los contenidos de la } \\
\text { asignatura sin intervención del alumnado. }\end{array}$ \\
\hline & $\begin{array}{l}\text { Lección } \\
\text { participativa }\end{array}$ & $\begin{array}{l}\text { El alumnado interviene, complementando o } \\
\text { dando feedback a la exposición del profesorado. }\end{array}$ \\
\hline \multirow[t]{5}{*}{$\begin{array}{l}\text { Clases } \\
\text { prácticas }\end{array}$} & $\begin{array}{l}\text { Estudio de casos } \\
\text { Resolución de problemas }\end{array}$ & $\begin{array}{l}\text { El alumnado resuelve cuestiones que se } \\
\text { plantean y que tienen como base una situación } \\
\text { concreta planteada por el profesorado. }\end{array}$ \\
\hline & Revisión bibliográfica & $\begin{array}{l}\text { Se propone la lectura o visualización de un } \\
\text { recurso como base del trabajo. }\end{array}$ \\
\hline & Simulación & $\begin{array}{l}\text { Se crea o representa una situación, que sirve } \\
\text { como fundamento para el trabajo práctico. }\end{array}$ \\
\hline & Trabajo cooperativo & $\begin{array}{l}\text { El trabajo en grupo se convierte en la herramienta } \\
\text { para trabajar en grupos de discusión, grupos } \\
\text { focales, debates, coloquios o foros. }\end{array}$ \\
\hline & $\begin{array}{l}\text { Aprendizaje Basado en } \\
\text { proyectos }\end{array}$ & $\begin{array}{l}\text { Se realizan propuestas de intervención } \\
\text { específica en el marco de la asignatura. }\end{array}$ \\
\hline
\end{tabular}

Apuntábamos que estas propuestas son solamente algunas de las posibilidades que pueden darse en el desarrollo de los diferentes tipos de sesión. En apartados posteriores, tendremos oportunidad de profundizar más en algunas de ellas.

\section{Los recursos para el aprendizaje}

El diseño de los procesos formativos responde a la naturaleza de la propia institución, optimizando la utilización de los recursos y posibilidades que ofrece la modalidad de enseñanza en línea

En cada una de las titulaciones, se pone a disposición del alumnado un conjunto de materiales en diferentes formatos (manual, documento multimedia y vídeos docentes), elaborados específicamente para cada asignatura y que sirven de fundamento para el desarrollo de la acción formativa.

Estos materiales son complementados con otros adicionales, propuestos a discreción de cada docente, y se integran pedagógicamente en el diseño de las actividades que estos proponen en el marco de cada asignatura. Por otro lado, se contempla la realización de tutorías, que pueden desarrollarse mediante diversos medios (correo electrónico, chat o videoconferencia), en ajuste a las necesidades y situaciones concretas que se suceden en cada una de las materias y en función del desarrollo de cada actividad.

\section{La plataforma Learning Management System (LMS)}

Los sistemas para la gestión del aprendizaje o learning management systems se están constituyendo en las últimas décadas como unas herramientas de valor incalculable para el desarrollo de modalidades online de enseñanza y aprendizaje (Cabero, Arancibia y Del Prete, 2019). Su potencial para crear entornos de aprendizaje colaborativo contribuye a la creación de escenarios donde diseñar acciones formativas de carácter diverso facilitando, asimismo, la puesta en práctica de metodologías y actividades casi incuantificables (Silva, García, Guzmán y Chaparro, 2016). Otra de sus ventajas reside en las múltiples funciones que ofrece tanto para la comunicación, como para el 
desempeño de los procesos de enseñanza y aprendizaje. Entre ellas, podemos destacar los apartados que sirven como módulos organizativos (presentación de las asignaturas y calendario), como repositorio de contenidos (guía didáctica, recursos y materiales para el estudiante), como canales comunicativos (últimas noticias, anuncios, envío de e-mail y foro) y como canalizadores de la formación (videoconferencias, actividades, blog, wiki, grupos y calificaciones).

\section{Herramientas de videoconferencia}

Las herramientas de videoconferencia cumplen una labor primordial para la puesta en marcha de las sesiones de trabajo síncronas (clases expositivas, clases prácticas y tutorías), constituyendo el eje fundamental de una interacción en tiempo real entre profesor y estudiante y entre el propio alumnado, gracias a su potencial para la deslocalización de la comunicación y el aprendizaje (Romero, 2019).

En el caso de estudio, la herramienta de videoconferencia está integrada en la plataforma LMS, pero tiene entidad independiente. Al margen de la comunicación profesor-estudiante y entre estudiantes mediada por vídeo (cámara) y audio (micrófono), esta herramienta incorpora funcionalidades diversas, que contribuyen a una experiencia formativa más inmersiva: un chat en tiempo real, posibilidad de compartir contenido en diferentes formatos (archivos PDF, presentaciones en PowerPoint e imágenes en formatos GIF, JPEG y PNG), realización de sondeos o interacción en grupos de trabajo.

El análisis de este contexto nos ofrece el marco sobre el que fundamentar la posterior disertación en torno a las propuestas metodológicas que fomentan el aprendizaje. Para ello, partimos de que la combinación de todas estas herramientas y recursos da lugar a procesos formativos de carácter interactivo que posibilitan la puesta en marcha de estrategias de aprendizaje diferentes por parte de los estudiantes, flexibilizando los modos en que cada uno de ellos desarrollan las competencias (Romero, San Martín y Peirats, 2018). La clave, en este sentido, es diseñar acciones formativas donde las metodologías y herramientas vayan en sintonía y favorezcan la adquisición de dichas competencias.

Las herramientas al servicio del aprendizaje: análisis del videoblog, el foro, la gamificación y el aprendizaje basado en proyectos en la formación en línea.

En el contexto de la modalidad en línea y el modelo académico de la institución descritos en apartados anteriores, se analizan a continuación algunas propuestas metodológicas, focalizando sobre su impacto sobre el aprendizaje colaborativo, el aprendizaje significativo, el aprendizaje autónomo y la construcción crítica del conocimiento por parte del alumnado.

Sin embargo, antes de proceder a este análisis, consideramos relevante definir estos tipos de aprendizaje y su vinculación a los entornos virtuales, a fin de contextualizar de un modo más pertinente el potencial de las herramientas en el proceso formativo.

\section{El impacto de los entornos virtuales en los diferentes tipos de aprendizaje}

Cabe comenzar exponiendo que, siendo conscientes de que la formación en línea se vincula con diferentes tipos y estrategias de aprendizaje, se ha realizado una selección de algunos de éstos, considerándolos los más afines a las herramientas y propuestas metodológicas en las que se va a profundizar. 
En primer lugar, las TIC tienen un potencial muy reseñable para el aprendizaje colaborativo, entendiendo por éste el que se desarrolla a través de un compromiso entre participantes y de un esfuerzo coordinado para dar respuesta a una tarea conjunta (Muhlenbrock, 1999). Implica, por tanto, una interdependencia positiva entre los distintos agentes (basada en un objetivo común), un fomento de la interacción (necesaria para la resolución del problema) y una responsabilidad individual (con uno mismo y con el grupo) (Barkley, Cross y Major, 2005; Scagnoli y Stephens, 2005). El aprendizaje colaborativo se presenta, así, como un complemento al trabajo individual, fundamentando su potencial en las relaciones con otros para el alcance de metas compartidas. Además, permiten el desarrollo de competencias interpersonales como el trabajo en equipo, la negociación o el liderazgo, vinculándose a la esfera más personal del alumnado. Bajo esta perspectiva, no cabe duda del potencial que tienen los contextos mediados por las tecnologías para el aprendizaje colaborativo, en la medida en que multiplican la creación de escenarios donde desarrollar procesos comunicativos y colaborativos. Estos nuevos ambientes, que como hemos apuntado anteriormente rompen barreras espaciales y temporales, son espacios donde la reflexión individual y el intercambio de ideas adquieren una nueva perspectiva, multiplicando las posibilidades para el desarrollo del proceso social de construcción colectiva del conocimiento (Coto, Collazos y Mora; 2016; Gil, 2019). Cabe destacar que, aunque en este espacio nos centramos en el aprendizaje colaborativo del alumnado, estas prácticas pueden constituir un elemento primordial para el desempeño del rol docente (Krichesky y Murillo, 2018), siendo un recurso valioso para la construcción de relaciones y conocimientos entre el propio profesorado.

En relación con el aprendizaje significativo, partimos de la idea de que es aquel que se fundamenta en la experiencia previa del sujeto, sirviendo esta como elemento básico para la interpretación de la información nueva a la que se enfrenta (Ausubel, Novak y Hanesian, 1991; Moreira, 2000). Se trata, por tanto, de un proceso personal por la cual cada persona interpreta o reinterpreta la realidad de un modo peculiar, integrándola en su estructura cognitiva y otorgándole su significado (Solé y Coll, 1993). Este tipo de aprendizaje, que se vincula de un modo inequívoco al proceso de aprendizaje autónomo y a la construcción crítica del conocimiento que analizaremos posteriormente, se potencia gracias a las Tecnologías de la Información y la Comunicación. En este sentido, las TIC sirven para motivar al alumnado en su interacción con la realidad, desarrollando habilidades de pensamiento crítico y creativo, integrando y reteniendo la información, y facilitando la comprensión de lo aprendido de una manera integral y dinámica (Esteve y Gisbert, 2011). Sin embargo, para que el aprendizaje sea realmente significativo, es imprescindible un buen diseño de la acción formativa por parte del profesorado. En este sentido, que se aborde el proceso desde el punto de partida del alumnado, se mantenga una comunicación constante y efectiva, se ofrezcan los contenidos en diversos formatos o se diseñen diferentes tipos de actividad, contribuyen a afrontar el desarrollo de las competencias desde diversos puntos de vista, facilitando así que el aprendizaje sea más significativo (Pablo, 2017).

En lo que respecta al aprendizaje autónomo, la naturaleza y concepción de los entornos virtuales ha conllevado la necesidad de que el alumnado adquiera un rol protagonista y activo en su aprendizaje, transformándose el proceso de enseñanza tradicional en una labor de "autogestión del conocimiento individual y el trabajo cooperativo" (Chica, 2010, p. 170). Así, se convierten en el máximo responsable del éxito de su proceso educativo, siendo claves factores como la motivación, la constancia o el esfuerzo para poder implementar procesos de autorregulación del aprendizaje de forma positiva. Consecuencia de este enfoque, se modifican los roles del proceso clásico de enseñanza-aprendizaje. La construcción del conocimiento pasa a ser responsabilidad del educando, quien asume la organización del trabajo adaptándola a su propio ritmo (García, Castañeda y Mansilla, 2018; Martínez Lirola, 2008), adquiriendo así las 
competencias y contenidos necesarios para poder enfrentarse a los distintos retos y problemáticas que les plantee la realidad (Sarmiento, 2012). En este sentido, es un factor importante el poseer conocimientos sobre las diferentes estrategias de aprendizaje y metodologías existentes, para así poder optar por aquellas que le sean más enriquecedoras para su proceso formativo. Además, al convertirse en el gestor de su propio aprendizaje, es preciso desarrollar un proceso de autoevaluación constante sobre las competencias a adquirir y los objetivos a alcanzar, pudiendo así reflexionar sobre los avances que se van realizando a la vez que se pone el foco en los aspectos que debe seguir trabajando (Muchiut et al., 2018). Por su parte, la labor del profesorado se orienta a una función de guía pedagógico y profesional (Peláez, 2009), siendo para ello preciso una correcta formación en el ámbito didáctico (Colen, Giné e Imbernón, 2006). El profesorado no desaparece del contexto formativo, pero debe reinventarse para responder a unas necesidades formativas en la que su papel como fuente de conocimiento no son precisas. De esta manera, el aprendizaje autónomo se convierte en máxima expresión de los procesos formativos e-learning, siendo el deseo de aprender el estímulo que lleva a los estudiantes a trabajar y esforzarse por organizar y gestionar de forma provechosa el tiempo para la consecución de las competencias estipuladas.

Una vez comentados los principales tipos de aprendizaje que se desarrollan en el entorno virtual descrito, pasamos a profundizar en varias propuestas metodológicas que sirven de ejemplo para conocer cómo y de qué forma se enseña y aprende en el contexto e-learning de la Universidad Internacional de Valencia.

\section{Propuestas metodológicas al servicio del aprendizaje}

Dentro de las múltiples opciones que se pueden abordar en los contextos de formación en línea, vamos a poner el foco en aquellas propuestas que, siendo diferenciadoras del modelo académico de la Universidad Internacional de Valencia, pueden tener una especial relevancia en el proceso de aprendizaje de sus estudiantes.

\section{Videoblog}

Los videoblogs, bajo la perspectiva educativa, permiten la comunicación unidireccional mediante la publicación de post audiovisuales en los que compartir información sobre los contenidos de las materias a las que se aplique y que permiten la interacción entre el alumnado al poder reflexionar, debatir y construir conocimiento en torno al mismo. El elemento distinto es el vídeo como canal de información, del cual se ha comprobado su eficiencia para el aprendizaje (Colomo y Aguilar, 2017). No obstante, conlleva un arduo trabajo previo de preparación de la entrada (planificar los contenidos, organizarlos, guionizar la información, revisión, etc.). Tras todo ello, se debe grabar, editar, publicar y hacer seguimiento de su impacto (Blanch, Betancort y Martínez, 2016). Respecto al espacio donde se alojan los vídeos, tener en cuenta que en dicha galería siempre se incluirán los vídeos ordenados de forma inversa a la temporalidad de su publicación. En definitiva, apostar por los videoblogs se debe a que es "una oportunidad pedagógica de discusión y construcción del conocimiento entre todos los agentes intervinientes en el proceso académico, acercándonos a un canal comunicativo más atractivo y lleno de posibilidades que permita un aprendizaje sin fronteras, global y holístico" (Colomo et al. 2018, p. 2887).

De cara a su introducción en los entornos virtuales de aprendizaje, el videoblog nos ofrece un resultado más atractivo y dinámico para su consumo (Colomo et al., 2018), favoreciendo su implementación en diferentes contextos formativos (Maceiras, Cancela y Goyanes, 2010). Puede resultarnos útil, entre otros aspectos, para abordar conceptos 
complejos mediante un lenguaje multimedia más motivador para los estudiantes, asemejándose más a sus hábitos de consumo comunicativo (Casero, Ortells y González, 2014). Se trata de una herramienta ya implementada en procesos formativos de educación superior (García, Melendo y Presol, 2013) de forma exitosa, siendo habitual el uso de material didáctico audiovisual en prácticamente todas las universidades del mundo (Maquilón, Mirete y Avilés, 2017). En el caso de la Universidad Internacional de Valencia, la apuesta por el uso del videoblog partía de la consigna de que no eran un fin en sí mismo (De la Horra, 2017), sino que su función era la de ser el medio que facilitara los procesos de enseñanza-aprendizaje y la adquisición de las competencias fijadas en el curriculum. De cara a su desarrollo, se propone una duración breve (5-10 minutos) y un enfoque novedoso para su difusión, adquiriendo el rol de material de apoyo o complementario para el proceso formativo.

Coincidiendo con muchas de las ventajas aportadas por García et al. (2013), enumeramos los principales beneficios que su uso ha supuesto para los procesos formativos desarrollados dentro del entorno virtual de la Universidad Internacional de Valencia:

- Rol activo del alumnado, con mayor protagonismo y autorregulación en su proceso de aprendizaje.

- Aumento de recursos motivadores y significativos para el alumnado, incorporando post de creación propia y otros materiales audiovisuales encontrados en la web.

- Fomento del trabajo en grupo y del aprendizaje colaborativo aprovechando las posibilidades técnicas de los videoblogs.

- Modifica el rol del profesorado, pasando a un papel de dinamizador y no como fuente de información principal.

- Desarrollo de las competencias digitales.

- Procesos de evaluación dinámicos, flexibles y con múltiples agentes, favoreciendo un feedback más enriquecedor.

\section{Foro}

El foro, entendido en el contexto formativo mediado por las tecnologías, supone un recurso con un potencial reseñable en la construcción de conocimiento crítico y para el aprendizaje colaborativo. Se suelen materializar en espacios virtuales en la que el alumnado intercambia opiniones, favoreciendo la interacción entre los diferentes agentes educativos en torno a una temática, habitualmente propuesta por el profesorado (Colomo, Gabarda y Rodríguez, 2018). Sin embargo, pueden producirse de forma espontánea a propuesta del alumnado, teniendo una capacidad destacable para la iniciativa de estos.

Se trata, por tanto, de un recurso cuya clave del éxito son la reflexión y la metacognición del estudiante (Basso, Bravo, Castro y Moraga, 2018), a partir de una situación de aprendizaje diseñada por el docente. El papel de éste, lejos de vincularse a una perspectiva tradicional de impartición teórica de contenidos, es el de facilitador y dinamizador del aprendizaje (Cuenca, 2015), convirtiéndose para el alumnado en un aprendizaje de conocimiento aplicado donde materializar la relación entre teoría práctica (Colomo et al., 2018). Aunque su aplicabilidad se extiende a cualquier contexto y etapa formativa, la utilización es cada vez más habitual en la educación superior (Gros y Adrián, 2016), dada su funcionalidad para la realización de actividades diversas (debate, intercambio de información o apoyo al proceso de aprendizaje, entre otras) y su utilidad para el aprendizaje colaborativo (Barrera, Montaño y Marín, 2017). 
Partiendo de estas aportaciones, y en base a las propuestas de Abawajy (2012), Garibay, Concari y Quintero (2013) y Fernández y Valverde (2014), los foros constituyen una herramienta básica en el modelo metodológico de la Universidad Internacional de Valencia. Su utilización como elemento formativo ha aportado una serie de beneficios para los procesos de enseñanza y aprendizaje:

- Considera al profesorado como un diseñador de la experiencia y un dinamizador del proceso.

- Convierte al alumnado en el responsable y potenciador del aprendizaje.

- Permite el trabajo colaborativo entre pares.

- Favorecen la vivencia de experiencias de aprendizaje más allá de las aulas físicas.

- Permiten un aprendizaje más significativo, al proporcionar más tiempo para la participación.

- Fomenta un aprendizaje autónomo y crítico.

- Promueve el aprendizaje a través de interacciones no mediadas por barreras espaciales y temporales.

- Posibilitan articular ideas desde diferentes fuentes de discusión.

- Facilita la construcción de conocimiento compartido.

- Favorece la cohesión grupal y la construcción de relaciones sociales.

- Favorece el desarrollo de las habilidades sociales.

- Fomenta el desarrollo de las competencias comunicativas, especialmente las de comunicación escrita.

- Permite expresar ideas con mayor libertad y facilidad.

\section{Aprendizaje Basado en Proyectos}

En el caso del Aprendizaje Basado en Proyectos (ABP O PBL, Proyect-Based Learning), nos encontramos ante una propuesta metodológica que tiene como fin favorecer procesos de aprendizajes significativos, adquiriendo el alumnado un rol protagonista y activo, teniendo para ello en cuenta aspectos claves del proceso formativo si queremos alcanzar una enseñanza holística, como los conocimientos previos, las estrategias de aprendizaje, el interés de los educandos o sus niveles de habilidad (Cuevas y Cívico, 2018). Entre las definiciones, podemos emplear la de García y Basilotta (2017), quienes hablan de "una modalidad de enseñanza y aprendizaje centrada en tareas, un proceso compartido de negociación entre los participantes, siendo su objetivo principal la obtención de un producto final" (p. 114). El eje central del ABP es configurar proyectos que den respuestas a las demandas, necesidades, innovaciones, dificultades 0 intereses partiendo de los contenidos que se estén abordando en los procesos de enseñanza. En dichos proyectos, se consideran diferentes fases a desarrollar de forma eficiente y rigurosa como la planificación, desarrollo y evaluación (Márquez y Jiménez, 2014). En este sentido, el foco se sitúa en que el alumnado sepa implementar procedimientos, destrezas y habilidades de forma autónoma para la realización de dichos proyectos (Martín y Rodríguez, 2015; Toledo y Sánchez, 2018). Respecto al profesorado, este desarrolla un papel de guía en el proceso educativo, siendo su principal cometido estimular al alumnado a aprender y descubrir (Lucendo, 2016). Estamos ante una metodología donde se promueve también un aprendizaje colaborativo, favoreciendo una construcción de conocimiento a partir de la interacción entre compañeros en base a los contenidos y experiencias que se aborden con la realización del proyecto (Sarceda, Seijás, Fernández y Fouce, 2016). Para lograrlo, es preciso una actitud de perseverancia, dedicación y esfuerzo en los principales actores del proceso formativo: alumnado y profesorado (Cascales, Carillo y Redondo, 2017). 
Pasamos a comentar las principales ventajas que la implementación del ABP ha tenido en el entorno virtual, coincidiendo en muchos casos con lo aportado por diferentes estudios (Barba, Sonlleva y García, 2018; Rekalde y García, 2015):

- Incrementa la motivación de los actores del proceso formativo.

- Aprendizaje asociado a la realidad, donde los proyectos surgen como forma innovadora de afrontar diferentes circunstancias ante las que nos podamos encontrar.

- Favorece la adquisición de destrezas en torno al diseño, la gestión, la selección, etc.

- Fomenta un desarrollo de competencias digitales respecto al aprendizaje y la tecnología.

Por todo lo expuesto, la aplicación del ABP en el contexto virtual de la Universidad Internacional de Valencia conlleva apostar por una herramienta que resulta positiva y enriquecedora para el aprendizaje, como han demostrado diferentes investigaciones (Moral, Villalustre y Neira, 2013; Vallina, Velasco, Aguilar y Rodríguez, 2018).

\section{Gamificación}

La introducción del juego como recurso didáctico no es un fenómeno nuevo. Lejos de eso, su utilización en los procesos formativos se ha ido consolidando durante las últimas décadas por su potencial para el fomento del aprendizaje significativo. En este sentido, el juego permite abordar, desde una perspectiva diferente (más lúdica y vivencial), el desarrollo de contenidos, destrezas y actitudes, redefiniendo la experiencia de aprendizaje. Por otro lado, la inmersión de las Tecnologías de la Información y la Comunicación en los diferentes contextos en que se suceden acciones formativas (formales o informales), ha potenciado, más si cabe, las posibilidades del juego, creando nuevos escenarios para los procesos de enseñanza y aprendizaje.

Sirva de ejemplo el uso cada vez más extendido de los videojuegos como herramienta didáctica. Se han constituido como instrumentos motivadores y de gran influencia a nivel cultural y social (Dorado y Gewerzc, 2017) y han contribuido a la consolidación de la gamificación como metodología para los procesos de enseñanza y aprendizaje (Díaz y Troyano, 2013). En este sentido, es importante tener en cuenta que la gamificación no es una actividad improvisada, sino que requiere repensar la acción formativa, incluyendo elementos de competición, cooperación, exploración y narración y que se orienta hacia la consecución de objetivos (Parente, 2016). Esto permite cumplir con algunas necesidades básicas del alumnado para el éxito del proceso formativo, como el reconocimiento, la recompensa, el logro, la competencia, la colaboración, la autoexpresión y el altruismo (Area y González, 2015). Su utilización, aunque inicialmente vinculada a etapas pre-obligatorias y obligatorias, es cada vez más habitual en educación superior (Cabezas y Alonso, 2015), donde van implementándose experiencias que contribuyen a diseñar nuevos modos de afrontar la formación universitaria. Y es que la gamificación, puede constituir un recurso de gran utilidad, no solo en el diseño de la acción formativa y en su desarrollo, sino para los propios procesos de evaluación, habiendo herramientas específicas para su implementación en ella (Marín, Vidal, Peirats y López, 2018).

En base a todo lo expuesto, y en consonancia con aportaciones como las de Contreras (2016), Oliva (2016) o Gil (2019), la gamificación aporta una serie de ventajas de valor incalculable para los procesos formativos:

- Permite el abordaje de competencias complejas desde una perspectiva lúdica. 
- Promueve el estímulo emocional, siendo este un impulsor del aprendizaje.

- Su estructuración facilita la organización del aprendizaje y proporciona feedback inmediato al estudiante sobre su proceso de aprendizaje.

- En este sentido, también facilita al docente la supervisión del proceso formativo, favoreciendo la evaluación continua.

- Los mecanismos de recompensa sirven como reforzadores del logro.

- Potencia la creatividad y la capacidad para la resolución de problemas.

- Aumenta la motivación hacia el proceso formativo, produciendo un aprendizaje más significativo.

- Fomenta el aprendizaje colectivo, favoreciendo dinámicas de trabajo para el grupo.

\section{Conclusiones}

La realidad está en constante evolución y la educación debe adaptarse para responder a las necesidades que la sociedad le demanda. Para ello, las tecnologías se convierten en el medio que permitirá a los enfoques pedagógicos y didácticos evolucionar dentro del contexto formativo, logrando así satisfacer con calidad y rigor los diferentes retos que vayan surgiendo en el ámbito educativo.

En este sentido, cualquier institución con fines formativos puede incorporar a su oferta académica los entornos virtuales como canal y medio de aprendizaje. La diferencia no radica en las herramientas, software o las múltiples acciones que se pueden llevar a cabo, ya que al final se trata del nivel de desarrollo de la plataforma y de aquellos elementos que sean precisos o no para el proceso de enseñanza y de aprendizaje. Lo que realmente es significativo, es el modelo académico y la intención educativa que persiga la institución, convirtiéndose las TIC en un medio y no un fin para la formación. De este modo, la Universidad Internacional de Valencia presenta una apuesta vanguardista por la tecnología como medio para poder desarrollar su propuesta pedagógica, la auténtica señal de identidad que la diferencia del resto de instituciones formativas. Por ello, es preciso partir siempre de un enfoque didáctico ante cualquier acto educativo que se vaya a implementar, pues las herramientas se van actualizando, transformando o, incluso, creando, pero el fin sigue perenne e inamovible pese a los cambios: a saber, adquirir las competencias y destrezas para enfrentarse a los distintos problemas y obstáculos que aparezcan en la realidad de forma eficiente y satisfactoria.

En esta realidad, los diferentes tipos de aprendizajes que se han venido desarrollando en el contexto de la educación tradicional, siguen estando presentes, aunque mediados por la tecnología. Es esta nueva realidad la que modifica algunos matices en sus concepciones sin que pierdan el sentido y esencia de estos. Así, a través de la tecnología, el aprendizaje colaborativo encuentra un instrumento para facilitar el trabajo en línea gracias a la deslocalización a nivel temporal y espacial; el aprendizaje significativo se potencia al abordar los contenidos desde diferentes formatos y enfoques didácticos, a la vez que se facilitan los procesos de evaluación previa y continua mediante las herramientas de seguimiento y control sobre el proceso formativo; y el aprendizaje autónomo encuentra un sinfín de posibilidades didácticas para la adquisición de las competencias, llevando a cabo procesos de gestión y organización del proceso de estudio de forma simplificada gracias a las facilidades que ofrece el entorno virtual.

Considerando que son múltiples las opciones y propuestas metodológicas que se pueden implementar, se han resaltado aquellas más significativas y alineadas con los fines didácticos y la concepción del acto educativo por parte de la Universidad Internacional de Valencia. Los videoblog representan a la perfección las posibilidades 
del mundo tecnológico, donde la conjunción de los medios visuales con la funcionalidad informativa de los blogs y su capacidad de difusión, permiten al alumnado adquirir conocimientos de una forma más motivadora y desarrollan competencias claves como la de aprender a aprender. Los foros facilitan un marco donde construir conocimiento de forma conjunta sin barreras temporales, fomentando el pensamiento crítico y la reflexión sobre los conocimientos y favoreciendo desarrollar la capacidad de argumentación escrita. En cuanto al Aprendizaje Basado en Proyectos, nos encontramos ante una realidad que ve aumentada su eficiencia gracias a la deslocalización y trabajar desde la red con múltiples recursos y posibilidades, teniendo como fin la consecución del proyecto y como límite los que el alumnado quiera estipular. La gamificación, por su parte, enriquece su potencialidad y posibilidades gracias a las tecnologías, donde los múltiples software y recursos dotan de mayor dinamismo y medios a las tareas generadas para adquirir los conocimientos mientras jugamos. Todas ellas son una simple muestra de cómo las metodologías se adaptan al entorno virtual de manera eficiente, incrementando o potenciando sus posibilidades didácticas y pedagógicas gracias a las tecnologías y la realidad del contexto online.

En definitiva, se trata de aprovechar los recursos y medios que las tecnologías ponen a nuestro alcance para desarrollar el proceso de enseñanza y de aprendizaje de la manera más enriquecedora posible.

\section{Referencias}

Abawajy, J (2012). Analysis of asynchronous online Discussion Forums for Collaborative Learning. International Journal of Education and Learning, 1(2), 11-21.

Area, M y González, CS (2015). De la enseñanza con libros de texto al aprendizaje en espacios online gamificados. Educatio Siglo XXI, 33(3), 15-38.

Armellini, A, \& Aiyegbayo, A (2010). Learning design and assessment with e-tivities. British Journal of Educational Technology, 41(6), 922-935.

Ausubel, D, Novak, J y Hanesian, H (1991). Psicología educativa: un punto de vista cognitivo. Ciudad de México: Trillas.

Barba, R, Sonlleva, M y García, N.j (2018). Presencia, participación y progreso: el aprendizaje basado en proyectos en la trayectoria de una muestra en formación. Revista Electrónica Interuniversitaria de Formación del Profesorado, 21(2), 13-25.

Barkley, E, Cross, KP, \& Major, CH (2005). Collaborative learning techniques: $A$ Handbook for college faculty. San Francisco: Jossey-Bass.
Barrera, R, Montaño, R y Marín, P (2017) Una intervención interactiva por medio de foros en el aprendizaje colaborativo. EDUTEC, Revista electrónica de Tecnología Educativa, 62, 73-82.

Basso, M, Bravo, M, Castro, A y Moraga, C (2018). Propuesta de modelo tecnológico para Flipped Classroom (T-FliC) en educación superior. Revista Electrónica Educare (Educare Electronic Journal), 22(2), 1-17.

Bernard, RM, Borokhovski, E, Schmid, RF, Tamim, RM, \& Abrami, PC (2014). A metaanalysis of blended learningand technology use in higher education: From the general to the applied. Journal of Computing in Higher Education, 26(1), 87-122.

Blanch, M, Betancort, S y Martínez, M (2016). El Videoblog en el Aula de Lengua y Literatura de Secundaria. Una propuesta práctica. REICE. Revista Iberoamericana sobre Calidad, Eficacia y Cambio en Educación, 14(3), 33-49.

Cabero, J, Arancibia, ML y del Prete, A (2019). Dominio técnico y didáctico del LMS Moodle en Educación Superior. Más allá de su uso funcional. Journal of New Approaches in Educational Research, 8(1), 27-35. 
Cabezas, SG y Alonso, C (2015). Gamificación en entornos educativos universitarios. Comunicación y pedagogía: Nuevas tecnologías y recursos didácticos, 281, 8691.

Cascales, A, Carrillo, ME y Redondo, AM (2017). ABP y tecnología en educación infantil. Píxel-Bit. Revista de Medios y Educación, 50, 201-209.

Casero, A, Ortells, S, y González, S (2014). Innovación educativa en el marco del EEES: el uso del videoblog como herramienta formativa en Periodismo. RIESED-Revista Internacional de Estudios sobre Sistemas Educativos, 2(3), 59-74.

Castaño, R, Jenaro, C y Flores, N (2017). Percepciones de estudiantes del Grado de Maestro sobre el proceso y resultados de la enseñanza semipresencial -Blended Learning-. Revista De Educación a Distancia, 52, 1-19.

Castaño, R, Poy, R, Tomşa, R, Flores, N, \& Jenaro, C (2015). Pre-service teachers'performance from teachers' perspective and vice versa: behaviors, attitudes and other associated variables. Teachers and Teaching: Theory and Practice, 21(7), 894-907.

Chica, FA (2010). Factores de la enseñanza que favorecen el aprendizaje autónomo en torno a las actividades de aprendizaje. Reflexiones Teológicas, 6, 167-195.

Colén, MT, Giné, N e Imbernón, F. (2006). La carpeta de aprendizaje del alumnado universitario. Barcelona: Octaedro.

Coll, C y Monereo, C (2008). Psicología de la Educación Virtual. Madrid: Morata.

Colomo, E y Aguilar, Al (2017). Píldoras formativas en la educación online: posibilidades y limitaciones. En J Ruiz, J Sánchez y E Sánchez (Edit.). Innovación docente y uso de las TIC en educación (pp. 1-10). Málaga: UMA Editorial.

Colomo, E, Motos, P, Aguilar, Al y Gabarda, V (2018). El videoblog como recurso didáctico en la metodología de formación online. En E. López, D. Cobos, A.H. Martín, L. Molina y A. Jaén (Eds.). Experiencias pedagógicas e innovación educativa. Aportaciones desde la praxis docente e investigadora (pp. 2878-2889). Barcelona: Octaedro.

Comas-Quinn, A (2011). Learning to teach online or learning to become an online teacher: An exploration of teachers' experiences in a blended learningcourse. ReCALL: Journal of Eurocall, 23(3), 218-232.
Contreras, RS (2016). Elementos de juego y motivación: reflexiones en torno a una experiencia que utiliza la gamificación en una asignatura de Grado para Game Designers. En RS Contreras y JL Eguia (Coords.). Gamificación en aulas universitarias (pp. 56-67). Bellaterra, España: Institut de la Comunicació, Universitat Autònoma de Barcelona.

Coto, M, Collazos, CA y Mora, S (2016). Modelo Colaborativo y Ubicuo para apoyar los procesos de enseñanza-aprendizaje a nivel Iberoamericano. RED-Revista de Educación a Distancia, 48(10), 1-30.

Cuenca, V (2015). The virtual forum as a strategy of teaching in higher education. Hamut'ay, 2(1), 23-31.

Cuevas, N y Cívico, A (2018). El desarrollo de las competencias básicas mediante el aprendizaje por proyectos. Harvad Deusto: Learning \& Pedagogics, 14, 30-35.

De la Horra, GI (2017). Realidad aumentada, una revolución educativa. Edmetic: revista de Educación Mediática y TIC, 6(1), 9-22.

Díaz, J y Troyano, Y (2013). El potencial de la gamificación aplicado al ámbito educativo. III Jornadas de Innovación Docente. Innovación Educativa: respuesta en tiempos de incertidumbre, Sevilla, España.

Dorado, S y Gewerc, A (2017). El profesorado español en la creación de materiales didácticos: Los videojuegos educativos. Digital Education Review, 31, 176-195.

Durán, M, Gutiérrez, I y Prendes, MP (2016). Propuesta de un modelo actualizado de competencia digital del profesorado: áreas, descriptores e indicadores. En R Roig (Ed.). Tecnología, innovación e investigación en los procesos de enseñanza-aprendizaje (pp. 1587-1598). Barcelona: Octaedro.

Durán, R y Estay, C (2016). Formación en buenas prácticas docentes para la educación virtual. RIED. Revista Iboeroamericana de Educación a Distancia, 19(1), 209-232.

Esteve, FM y Gisbert, M (2011). El nuevo paradigma de aprendizaje y las nuevas tecnologías. Revista de Docencia Universitaria, REDU, 9(3), 55-73.

Falco, M (2017). Reconsiderando las prácticas educativas: TICs en el proceso de enseñanza-aprendizaje. Tendencias pedagógicas, 29, 59-76. 
Fernández, M y Valverde, J (2014). Comunidades de práctica: un modelo de intervención desde el aprendizaje colaborativo en entornos virtuales. Comunicar, XXI, 42, 97-105.

García, A y Basilotta, V (2017). Aprendizaje basado en proyectos (ABP): evaluación desde la perspectiva de alumnos de Educación Primaria. Revista de Investigación Educativa, 35(1), 113-131.

García, B, Luna, E, Ponce, S, Cisneros, EJ, Cordero, G. y Espinosa, Y. (2018). Las competencias docentes en entornos virtuales: un modelo para su evaluación. RIED. Revista Iboeroamericana de Educación a Distancia, 21(1), 343-365. doi: https://doi.org/10.5944/ried.21.1.18816

García, C., Castañeda, E. y Mansilla, J.M. (2018). Experiencia de innovación en el aula desde la autorregulación y los estilos de aprendizaje. Tendencias Pedagógicas, 31, 137-148.

García, E., Melendo, L. y Presol, A. (2013). Recursos audiovisuales en la docencia a nivel universitaria. El uso del videoblog como herramienta de comunicación. Historia y Comunicación Social, 18,159172.

doi: https://doi.org/10.5209/rev_hics.2013.v18.4 4322

Garibay, T., Concari, B. y Quintero, B. (2013). Desarrollo del aprendizaje colaborativo empleando tareas mediadas por foros virtuales. Etic@net Revista Científica Electrónica de Educación y Comunicación en la Sociedad del Conocimiento, 2(13), 273-300.

Gil, J. (2019). Interconectados apostando por la construcción colectiva del conocimiento. Aprendizaje móvil en educación infantil y primaria. Pixel-BIT Revista de Medios y Educación, 54, 185-203. doi: https://doi.org/10.12795/pixelbit.2019.i54.1 0

Gros, B. y Adrián, M. (2016). Estudio sobre el uso de los foros virtuales para favorecer las actividades colaborativas en la enseñanza superior. Education In The Knowledge Society (EKS), 5(1), 1-11.

Johnson, L., Adams, S., Cummins, M., Estrada, V., Freeman, A. y Hall, C. (2016). NMC Informe Horizon 2016 Edición Superior de Educación. Austin, Texas: The New Media Consortium.
Jonassen, D., Howland, J., Marra, R.M. y Crismond, D. (2008). Meaningful Learning with Technology. Upper Saddle River, N.J: Pearson.

Krichesky, G. J. y Murillo F. J. (2018). La colaboración docente como factor de aprendizaje y promotor de mejora. Un estudio de casos. Educación XX1, 21(1), 135-156.

Lucendo, AL (2016). Propuesta de metodología docente para la adaptación de materias de geografía descriptiva al EEES. El caso de la asignatura de Geografía de Europa. Didáctica Geográfica, 17, 79-100.

Maceiras, R., Cancela, Á. y Goyanes, V. (2010). Aplicación de nuevas tecnologías en la docencia universitaria. Formación Universitaria, 3(1), 21-26.

Maquilón, J.J., Mirete, A.B., y Avilés, M. (2017). La Realidad Aumentada (RA). Recursos y propuestas para la innovación educativa. Revista Electrónica Interuniversitaria de Formación del Profesorado, 20(2), 183-$203 . \quad$ doi: http://dx.doi.org/10.6018/reifop.20.1.29097 1

Marín, D., Vidal, M. I., Peirats, J., y López, M. (2018). Gamificación en la evaluación del aprendizaje: valoración del uso de Kahoot! En REDINE (Ed.). Innovative strategies for Higher Education in Spain (pp. 8-17). Eindhoven, NL: Adaya Press.

Márquez, E. y Jiménez, M.L. (2014). Projectbased learning in virtual environments: a case study of a university teaching experience. RUSC. Revista de Universidad y Sociedad del Conocimiento, 11(1), 76-90.

Martín, A. y Rodríguez, S. (2015). Motivación en alumnos de Primaria en aulas con metodología basada en proyectos. Revista de Estudios e Investigación en Psicología y Educación, 0(1), 58-62.

Martínez Lirola, M. (2008). Una propuesta de evaluación en el EEES: el uso del portfolio en una clase de idiomas. Porta Linguarum, 9, 23-34.

Moral, M.E., Villalustre, L. y Neira, M.R. (2013). Oportunidades de las TIC para la innovación educativa en las escuelas rurales de Asturias. Aula Abierta, 42(1), 6167. doi: https://doi.org/10.1016/S02102773(14)70010-1 
Moreira, M. (2000). Aprendizaje significativo: teoría y práctica. Madrid: Visor.

Muchiut, A.F., Zapata, R.B., Comba, A., Mari, M., Torres, N., Pellizardi, J. y Segovia, A.P. (2018). Neurodidáctica y autorregulación del aprendizaje, un camino de la teoría a la práctica. Revista Iberoamericana de Educación, 78(1), 205-219.

Muhlenbrock, M. (1999). A system for Analyzing Collaborative problem solving. Recuperado de http://citeseer.nj.nec.com/410548.html

Oliva, H.A. (2016). La gamificación como estrategia metodológica en el contexto educativo universitario. Realidad $y$ Reflexión, 16(44), 29-47.

Orozco, G.H., Cabezas. M., Martínez, F. y Alonso, M. (2016). Validación de un cuestionario para determinar las Competencias Digitales del profesorado universitario y la Aceptación de las TIC en su práctica docente. En R. Roig (Ed.). Tecnología, innovación e investigación en los procesos de enseñanza-aprendizaje (pp. 983-993). Barcelona: Octaedro.

Owens, T. (2012).Hittingthenailonthehead:The importance of specific staff development for effective blended learning. Innovations in Education and Teaching International, 49(4), 389-400. doi: https://doi.org/1080/14703297.2012.72887 7.

Pablo, G. (2017). Factores que favorecen la presencia docente en entornos virtuales de aprendizaje. Tendencias pedagógicas, 29, 43-58. doi: http://dx.doi.org/10.15366/tp2017.29.001

Parente, D. (2016). Gamificación en la educación. En R.S. Contreras y J.L. Eguia (Coords.). Gamificación en aulas universitarias (pp. 10-25). Bellaterra, España: Institut de la Comunicació, Universitat Autònoma de Barcelona.

Rekalde, I. y García, J. (2015). El aprendizaje basado en proyectos: un constante desafío. Innovación educativa, 25, 219-234.

Rodríguez, N. (2014). Fundamentos del proceso educativo a distancia: enseñanza, aprendizaje y evaluación. RIED. Revista Iboeroamericana de Educación a Distancia, 17(2), 75-93

Rodríguez, R.M. (2003). Reaprender a enseñar: Una experiencia de formación para la mejora continua de la docencia universitaria. Revista Interuniversitaria de Formación del Profesorado, 17(2), 79-84.
Rodríguez, R.M. (2010). El impacto de las TIC en la transformación de la enseñanza universitaria: repensar los modelos de enseñanza y aprendizaje. Revista Teoría de la Educación: Educación y Cultura en la Sociedad de la Información, 11(1), 32-68.

Romero, M.M., San Martín, Á. y Peirats, J. (2018). Diferencias de sexo en estrategias de aprendizaje de estudiantes online. Innoeduca. International Journal of Technology and Educational Innovation, 4(2), 114-126. doi: http://dx.doi.org/10.24310/innoeduca.2018. v4i2.4940

Romero, J. (2019). La videollamada como recurso tecnológico para la Educación Superior a Distancia. Red de Investigación Educativa, 11(1), 56 - 61.

Ruiz, J., Sánchez, J. y Gómez, M. (2013). Entornos personales de aprendizaje: estado de la situación en la Facultad de Ciencias de la Educación de la Universidad de Málaga. Píxel-Bit. Revista de Medios y Educación, 42, 171-181.

Ruiz, M. (2016). La universidad ante los nuevos escenarios virtuales de aprendizaje. En M.A. Santos Rego (Coord.). Sociedad del conocimiento: aprendizaje e innovación en la universidad (pp. 91-114). Madrid: Biblioteca Nueva.

Sarceda, M. C., Seijas, S. M., Fernández, V. y Fouce, D. (2016). El trabajo por proyectos en Educación Infantil: aproximación teórica y práctica. Redalei. Revista Latinoamericana de Educación Infantil, 4(3), 159-176.

Sarmiento, L.A. (2012). Ética Autónoma para un Aprendizaje Autónomo. Revista Quaestiones Disputatae, 11, 19-32.

Scagnoli, N. y Stephens, M. (2005). Collaborative learning strategies in online education. Online Conference for Teaching and Learning (IOC2005), Illinois, Estados Unidos.

Silva, J.E. y Romero, M.R. (2014). La virtualidad una oportunidad para innovar en educación: un modelo para el diseño de entornos virtuales de aprendizaje. Didasc@lia: Didáctica y Educación, V(1), 1-23.

Silva, M., García, T., Guzmán, T. y Chaparro, R. (2016). Estudio de herramientas Moodle para desarrollar habilidades del siglo XXI. Campus Virtuales, 5(2), 58-69. 
Solé, I. y Coll, C. (1993). Los profesores y la concepción constructivista. En C. Coll, E. Martín, T. Mauri, M. Miras, J. Onrubia e I. Solé (Eds.). El constructivismo en el aula (pp. 7-23). Barcelona: Graó.

Toledo, P. y Sánchez, J.M. (2018). Aprendizaje basado en proyectos: una experiencia universitaria. Profesorado. Revista de currículum y formación de profesorado, 22(2), 471-491.

Unesco (2012). Aprendizaje móvil para docentes en américa latina. Análisis del potencial de las tecnologías móviles para apoyar a los docentes y mejorar sus prácticas. París: Unesco.

Vallina, A., Velasco, I., Aguilar, Á.I. y Rodríguez, Á.D. (2018). Aplicación en la asignatura de Geografía de Asia y África de la metodología didáctica de aprendizaje basado en proyectos. Revista de Investigación Educativa de la Escuela de Graduados en Educación, 9(17), 49-60.

Vázquez, E. y Sevillano, M.L. (Eds.) (2015). Dispositivos digitales móviles en educación. Madrid: Narcea.

Westbrook, V. (2006). The virtual learning future. Teaching in Higher Education, 11(4), 471482. 